\title{
Continuous time random walks for the evolution of Lagrangian velocities
}

\author{
Marco Dentz, ${ }^{1,2,{ }^{*}}$ Peter K. Kang, ${ }^{3}$ Alessandro Comolli, ${ }^{1,2,4}$ \\ Tanguy Le Borgne, ${ }^{5}$ and Daniel R. Lester 6 \\ ${ }^{1}$ Spanish National Research Council (IDAEA-CSIC), 08034 Barcelona, Spain \\ ${ }^{2}$ Hydrogeology Group (CSIC-UPC), 08034 Barcelona, Spain \\ ${ }^{3}$ Korea Institute of Science and Technology, Seoul 136-791, Republic of Korea \\ ${ }^{4}$ Department of Civil Engineering, Technical University of Catalonia, 08034 Barcelona, Spain \\ ${ }^{5}$ Université de Rennes 1, CNRS, Geosciences Rennes, UMR No. 6118, Rennes, France \\ ${ }^{6}$ School of Civil, Environmental and Chemical Engineering, RMIT University, \\ Melbourne, Victoria 3000, Australia
}

(Received 6 August 2016; published 23 November 2016)

\begin{abstract}
We develop a continuous time random walk (CTRW) approach for the evolution of Lagrangian velocities in steady heterogeneous flows based on a stochastic relaxation process for the streamwise particle velocities. This approach describes the persistence of velocities over a characteristic spatial scale, unlike classical random walk methods, which model the persistence over a characteristic time scale. We first establish the relation between Eulerian and Lagrangian velocities for both equidistant and isochrone sampling along streamlines, under transient and stationary conditions. Based on this, we develop a space-continuous CTRW approach for the spatial and temporal dynamics of Lagrangian velocities. While classical CTRW formulations have nonstationary Lagrangian velocity statistics, the proposed approach quantifies the evolution of the Lagrangian velocity statistics under both stationary and nonstationary conditions. We provide explicit expressions for the Lagrangian velocity statistics and determine the behaviors of the mean particle velocity, velocity covariance, and particle dispersion. We find strong Lagrangian correlation and anomalous dispersion for velocity distributions that are tailed toward low velocities as well as marked differences depending on the initial conditions. The developed CTRW approach predicts the Lagrangian particle dynamics from an arbitrary initial condition based on the Eulerian velocity distribution and a characteristic correlation scale.
\end{abstract}

DOI: 10.1103/PhysRevFluids.1.074004

\section{INTRODUCTION}

The dynamics of Lagrangian velocities in fluid flows are fundamental for the understanding of tracer dispersion, anomalous transport behaviors (but also pair dispersion and intermittent particle velocity and acceleration time series), and fluid stretching and mixing. A classical stochastic viewpoint on particle velocities in heterogeneous flows is their representation in terms of Langevin models for the particle velocities [1], which account for temporal persistence, and the random nature of velocity through a Gaussian white noise. Such approaches assume that velocity time series form a Markov process when measured isochronically along a particle trajectory [2].

The observation of intermittency in Lagrangian velocity and acceleration time series in steady heterogeneous flow [3-5] questions the assumptions that underly the representation of Lagrangian velocity in terms of a classical random walk. Observed intermittency patterns manifest themselves in long episodes of low velocities and relatively short episodes of high velocity. This indicates an organizational principle of Lagrangian velocities that is different from the one implied in a temporal Markov processes, which assumes that velocities are persistent for a constant time interval of charac-

*marco.dentz@csic.es 
teristic duration $\tau_{c}$. The observed intermittency for flow through disordered media [3-5] suggests that particle velocities are persistent along a characteristic length scale $\ell_{c}$ along streamlines. Approaches that model particle velocities as Markov processes in space assign to particle transitions a random transition time, which is given kinematically by the transition distance divided by the transition velocity. Thus, such approaches are termed continuous time random walks (CTRWs) [6-9]. They are different from classical random walk approaches, which employ a constant discrete transition time.

Particle motion and particle dispersion have been shown to follow CTRW dynamics for flow through pore and Darcy-scale heterogeneous porous and fractured media [10-18], as well as turbulent flows $[19,20]$. While the CTRW provides an efficient framework for the quantification of anomalous dispersion and intermittency in heterogeneous flows, some key questions remain open regarding the relation of particle velocities and Eulerian flow statistics, and the stationarity of Lagrangian velocity statistics.

In classical CTRW formulations, particle velocities are nonstationary. This means, for example, that the velocity mean and covariance evolve in time. This property is termed aging [21]. However, for steady divergence-free random flows, such as flow through porous media, it has been found that particle velocities may in fact be stationary [22]; specifically the Lagrangian mean velocity may be independent of time. Furthermore, it has been found for flow through random fracture networks that the Lagrangian velocity statistics depends on the initial particle distribution [23-25]. Hence, in general, Lagrangian velocities are expected to evolve from an arbitrary initial distribution toward an asymptotic stationary distribution. Quantifying this property, which is not described in current CTRW frameworks, is critical for upscaling transport dynamics through disordered media, whose transport properties are sensitive to the initial velocity distribution.

In this paper, we study the evolution of Lagrangian velocities and their relation with the Eulerian velocity statistics. To this end, we discuss in the following section the concepts of Lagrangian velocities determined isochronically and equidistantly along streamlines and their relation to the Eulerian velocity. Furthermore, we recall some fundamental properties that elucidate the conditions under which they are transient or stationary. In Sec. III we derive the Lagrangian velocity statistics in the classical CTRW and develop a Markov-chain CTRW approach that models the evolution of equidistant streamwise Lagrangian velocities as a stochastic relaxation process. In this framework, in Sec. IV we derive explicit expressions for the one- and two-point statistics of Lagrangian velocities and analyze the evolution of the mean particle velocity, its covariance, and particle dispersion.

\section{LAGRANGIAN VELOCITIES}

We consider purely advective transport in a heterogeneous stationary velocity field $\mathbf{u}(\mathbf{x})$. Particle trajectories are described by the advection equation

$$
\frac{d \mathbf{x}(t, \mathbf{a})}{d t}=\mathbf{v}(t, \mathbf{a})
$$

where $\mathbf{v}(t, \mathbf{a})=\mathbf{u}[\mathbf{x}(t, \mathbf{a})]$ denotes the Lagrangian particle velocity. The initial particle position is given by $\mathbf{x}(t=0, \mathbf{a})=\mathbf{a}$. The particle motion can be described in terms of the distance $s(t, \mathbf{a})$ traveled along a trajectory, which is given by

$$
\frac{d s(t, \mathbf{a})}{d t}=v_{t}(t, \mathbf{a}), \quad \frac{d t(s, \mathbf{a})}{d s}=\frac{1}{v_{s}(s, \mathbf{a})} .
$$

We define the t-Lagrangian particle velocity as $v_{t}(t, \mathbf{a})=|\mathbf{v}(t, \mathbf{a})|$ and the s-Lagrangian velocity as $v_{s}(s, \mathbf{a})=v_{t}[t(s, \mathbf{a}), \mathbf{a}]$. The initial velocities are denoted by $v_{0}(\mathbf{a}) \equiv v_{t}(t=0, \mathbf{a}) \equiv v_{s}(s=0, \mathbf{a})$.

The absolute Eulerian velocities are defined by $v_{e}(\mathbf{x})=|\mathbf{u}(\mathbf{x})|$. Their probability density function (PDF) is defined through spatial sampling as

$$
p_{e}(v)=\lim _{V \rightarrow \infty} \frac{1}{V} \int_{\Omega} d \mathbf{x} \delta\left[v-v_{e}(\mathbf{x})\right],
$$


where $\Omega$ is the sampling domain and $V$ its volume. We assume here Eulerian ergodicity, which means that spatial sampling is equal to ensemble sampling such that

$$
p_{e}(v)=\overline{\delta\left[v-v_{e}(\mathbf{x})\right]},
$$

where the overbar denotes the ensemble average. In the following, we discuss the t-Lagrangian velocities $v_{t}(t, \mathbf{a})$, which are sampled isochronally along particle trajectories, and the s-Lagrangian velocities $v_{s}(s, \mathbf{a})$, which are sampled equidistantly along particle trajectories. Here and in the following, we assume both Eulerian and Lagrangian ergodicity. As outlined below, Lagrangian ergodicity implies that the statistics of particle velocities $v_{t}(t, \mathbf{a})$ sampled in time along a trajectory coincide with the statistics obtained by sampling between particles.

\section{A. Steady Lagrangian velocity distributions}

The PDF of the t-Lagrangian velocity is defined by isochrone sampling along a particle trajectory as

$$
p_{t}(v, \mathbf{a})=\lim _{T \rightarrow \infty} \frac{1}{T} \int_{0}^{T} d t \delta\left[v-v_{t}(t, \mathbf{a})\right] .
$$

Under Lagrangian ergodic conditions, it is independent of the initial particle position a and equal to the average over an ensemble of particles

$$
p_{t}(v)=\lim _{V_{0} \rightarrow \infty} \frac{1}{V_{0}} \int_{\Omega_{0}} d \mathbf{a} \delta\left[v-v_{t}(t, \mathbf{a})\right] .
$$

The latter is equal to the Eulerian velocity PDF due to volume conservation

$$
p_{t}(v)=\lim _{V_{0} \rightarrow \infty} \frac{1}{V_{0}} \int_{\Omega(t)} d \mathbf{x} \delta\left[v-v_{e}(\mathbf{x})\right] \equiv p_{e}(v),
$$

which can be seen by performing a change of variables according to the flow map $\mathbf{a} \rightarrow \mathbf{x}(t, \mathbf{a})$ and recalling that the Jacobian is one due to the incompressibility of the flow field.

The PDF of the s-Lagrangian velocity is defined in analogy to (5) by equidistant sampling along a particle trajectory as

$$
p_{s}(v, \mathbf{a})=\lim _{L \rightarrow \infty} \frac{1}{L} \int_{0}^{L} d s \delta\left[v-v_{s}(s, \mathbf{a})\right] .
$$

Changing variables under the integral according to the kinematic relationship (2) between $t$ and $s$ gives immediately

$$
p_{s}(v, \mathbf{a})=\frac{v p_{t}(v, \mathbf{a})}{\left\langle v_{t}\right\rangle},
$$

which means that the s-Lagrangian velocity PDF is equal to the flux-weighted t-Lagrangian velocity PDF. This can also be understood intuitively by the fact that isochrone sampling as expressed through $p_{t}(v)$ gives a higher weight to low velocities because particles spend more time at low velocities, while equidistant sampling assigns the same weight to high and low velocities.

Under conditions of Lagrangian ergodicity, we thus have that (i) $p_{s}(v, \mathbf{a})=p_{s}(v)$ is independent of the particle trajectory and equal to the average over an ensemble of particles and (ii) the s-Lagrangian velocity PDF is related to the Eulerian velocity PDF through flux weighting as

$$
p_{s}(v)=\frac{v p_{e}(v)}{\left\langle v_{e}\right\rangle} .
$$

The latter establishes the relation between s-Lagrangian and Eulerian velocity distributions. 


\section{B. Transient Lagrangian velocity distributions}

In the previous sections, we considered the PDFs of $\mathrm{t}$ - and s-Lagrangian velocities under stationary conditions. Here we focus on their transient counterparts, which are defined through a spatial average over an arbitrary normalized initial particle distribution $\rho(\mathbf{a})$.

The PDF of $\mathrm{t}$-Lagrangian velocities then is defined by

$$
\hat{p}_{t}(v, t)=\int d \mathbf{a} \rho(\mathbf{a}) \delta\left[v-v_{t}(t, \mathbf{a})\right] .
$$

Its temporal average is given by

$$
\lim _{T \rightarrow \infty} \frac{1}{T} \int_{0}^{T} d t \hat{p}_{t}(v, t)=p_{t}(v)=p_{e}(v)
$$

and thus its steady-state PDF is of course given by the Eulerian velocity PDF. In analogy, we consider the PDF of s-Lagrangian velocities for an arbitrary initial PDF

$$
\hat{p}_{s}(v, s)=\int d \mathbf{a} \rho(\mathbf{a}) \delta\left[v-v_{s}(s, \mathbf{a})\right] .
$$

Its average along a streamline is given by

$$
\lim _{L \rightarrow \infty} \frac{1}{L} \int_{0}^{L} d s \hat{p}_{t}(v, s)=p_{s}(v)=\frac{v p_{e}(v)}{\left\langle v_{e}\right\rangle} .
$$

The initial conditions for both the t-Lagrangian and s-Lagrangian velocity PDFs are identical,

$$
\hat{p}(v, s=0)=\hat{p}(v, t=0)=p_{0}(v) .
$$

Thus, as their respective steady-state PDFs are different, either one or both of them need to evolve, depending on whether the initial PDF is the flux-weighted Eulerian PDF [the steady-state PDF for $\hat{p}_{s}(v, s)$ ], the Eulerian PDF [the steady-state PDF for $\left.\hat{p}_{t}(v, t)\right]$, or neither of the two.

The initial velocity PDF depends on the particle injection mode. For example, a uniform in space particle injection corresponds here to an initial velocity PDF equal to the Eulerian PDF

$$
p_{0}(v)=\lim _{V_{0} \rightarrow \infty} \frac{1}{V_{0}} \int_{\Omega_{0}} d \mathbf{a} \delta\left[v-v_{0}(\mathbf{a})\right] \equiv p_{e}(v)
$$

because of Eulerian ergodicity. As this initial distribution is equal to the asymptotic steady tLagrangian velocity distribution, the $\hat{p}_{t}(v, t)=p_{e}(v)$ is independent of time for this initial injection condition, while the $\hat{p}_{s}(v)$ evolves with distance from the injection.

A flux-weighted particle injection mode corresponds to an initial velocity PDF equal to the flux-weighted Eulerian PDF

$$
p_{0}(v)=\lim _{V_{0} \rightarrow \infty} \frac{1}{V_{0}} \int_{\Omega_{0}} d \mathbf{a} \frac{v_{0}(\mathbf{a})}{\left\langle v_{e}\right\rangle} \delta\left[v-v_{0}(\mathbf{a})\right] \equiv \frac{v p_{e}(v)}{\left\langle v_{e}\right\rangle}
$$

again because of Eulerian ergodicity. As this initial distribution is equal to the asymptotic steady s-Lagrangian velocity distribution, $\hat{p}_{s}(v, s) \equiv p_{s}(v)$ is independent of $s$ for this initial injection condition, while $\hat{p}_{t}(v, t)$ evolves with time.

A pointlike injection at the initial position $\mathbf{x}(t=0 \mid \mathbf{a})=\mathbf{a}$ corresponds to the $\delta$ initial PDF

$$
p_{0}(v)=\delta\left[v-v_{0}(\mathbf{a})\right] .
$$

For this initial condition, both the t-Lagrangian and s-Lagrangian velocities are unsteady.

The evolution of Lagrangian velocities may be very slow and thus have a strong impact on the transport dynamics. This is the case in particular for heavy-tailed (toward low velocities) velocity distributions that induce long-range temporal correlations of particle velocities. In the following we study the quantification of the evolution of the Lagrangian velocity PDFs in a Markov model in $s$, which means the distance along a streamline. 


\section{Lagrangian velocity series}

We have established that the Lagrangian velocity PDFs evolve with travel time or travel distance along a streamline, unless the initial velocity distribution coincides with the respective steady-state PDF. In order to quantify this evolution, we need to model the Lagrangian velocity series. As mentioned in the Introduction, a classical approach is to model the t-Lagrangian velocity as a Markov process, based on the assumption or observation that velocities decorrelate on a characteristic time scale $\tau_{c}$. Thus, the equations of motion (2) may be discretized isochronically as

$$
t_{n+1}=t_{n}+\Delta t, \quad s\left(t_{n+1}\right)=s\left(t_{n}\right)+v_{t}\left(t_{n}\right) \Delta t .
$$

Velocity time series have been modeled by Langevin equations of the type [1]

$$
\tilde{v}_{t}\left(t_{n+1}\right)=\tilde{v}_{t}\left(t_{n}\right)-\frac{\Delta t}{\tau_{c}} \tilde{v}_{t}\left(t_{n}\right)+\sqrt{\frac{2 \sigma_{v}^{2} \Delta t}{\tau_{c}}} \xi\left(t_{n}\right),
$$

which describes an Ornstein-Uhlenbeck process for the velocity fluctuation $\tilde{v}_{t}\left(t_{n}\right)=v_{t}\left(t_{n}\right)-\left\langle v_{t}\right\rangle$. The noise $\xi\left(t_{n}\right)$ is Gaussian distributed with zero mean and unit variance. The steady-state distribution $p_{t}(v)$ here is Gaussian with mean $\left\langle v_{t}\right\rangle$ and variance $\sigma_{v}^{2}$. Under stationary conditions, the velocity correlation is exponential with correlation time $\tau_{c}$. Evidently, this modeling framework is limited to Gaussian statistics and short-range correlation in time.

Here we consider a different modeling approach. As pointed out in the Introduction, there has been ample evidence that particle motion in the flow through random porous and fractured media may be quantified by a CTRW [9]. In fact, as a consequence of the existence of a spatial correlation length scale for, e.g., the hydraulic conductivity or pore structure, flow velocities are expected to vary over a characteristic length scale $\ell_{c}$. This implies for t-Lagrangian velocities that a given velocity $v_{t}$ persists for a duration of $\ell_{c} / v_{t}$ and specifically that small velocities are more strongly correlated in time than high velocities $[26,27]$. This characteristic can explain intermittency in velocity and acceleration time series [3-5]. The existence of a characteristic length scale $\ell_{c}$ suggests discretizing the equations of motion (2) along a particle trajectory equidistantly such that

$$
s_{n+1}=s_{n}+\Delta s, \quad t\left(s_{n}\right)=t\left(s_{n}\right)+\frac{\Delta s}{v_{s}\left(s_{n}\right)} .
$$

Here the s-Lagrangian velocity series $v_{s}\left(s_{n}\right)$ is modeled as Markov process, which renders the equations of motion (21) a CTRW. In the following, we analyze the evolution of the Lagrangian velocity statistics in the setup of a classical CTRW characterized by independent s-Lagrangian velocities and a CTRW in which the velocity series is modeled as a Markov process through a stochastic relaxation.

\section{CONTINUOUS TIME RANDOM WALK}

We study now the evolution of space and time Lagrangian velocities in the CTRW framework. The classical approach assigns to each particle transition a transit time $\tau$ that is sampled at each step from its PDF $\psi(t)$. The transition times are related to the characteristic transition length $\ell_{c}$ and s-Lagrangian velocities $v_{s}$ as $\tau=\ell_{c} / v_{s}$. Thus, independence of subsequent transit times implies independence of subsequent s-Lagrangian velocities. In the following, we first consider the evolution of t-Lagrangian velocities in this classical CTRW formulation. The velocity statistics turn out to be nonstationary at finite times. We then study a CTRW formulation that is based on a Markov process for the s-Lagrangian velocities that allows for an evolution of both the s- and t-Lagrangian velocities. 


\section{A. Independent s-Lagrangian velocities}

Particle motion along a particle trajectory is quantified in the framework of a classical CTRW by the recursion relations

$$
s_{n+1}=s_{n}+\ell_{c}, \quad t_{n+1}=t_{n}+\tau_{n}
$$

where the transition length $\ell_{c}$ denotes a characteristic length scale on which streamwise velocities $v_{n} \equiv v_{s}\left(s_{n}\right)$ decorrelate. In this framework, the particle velocity is constant between turning points. Thus, the transition times $\tau_{n}=\ell_{c} / v_{n}$ are independent and identically distributed random variables. Their PDF is given by $\psi(\tau)$. It is related to the distributions of s-Lagrangian and Eulerian velocities by

$$
\psi(\tau)=\frac{\ell_{c}}{\tau^{2}} p_{s}\left(\ell_{c} / \tau\right)=\frac{\ell_{c} \tau_{v}}{\tau^{3}} p_{e}\left(\ell_{c} / \tau\right),
$$

where we defined the advection time scale $\tau_{v}=\ell_{c} /\left\langle v_{e}\right\rangle$. Note that the mean transit time $\langle\tau\rangle=\tau_{v}$ is equal to the characteristic advection time.

In this framework, the t-Lagragian velocity is given by

$$
v_{t}(t)=v_{n_{t}},
$$

where the renewal process $n_{t}=\sup \left(n \mid t_{n} \leqslant t\right)$ denotes the number of steps needed to arrive at time $t$. The PDF of the t-Lagrangian velocity is given by

$$
\hat{p}_{t}(v, t)=\left\langle\delta\left(v-v_{n_{t}}\right)\right\rangle .
$$

This expression can be expanded as

$$
\hat{p}_{t}(v, t)=p_{s}(v) \int_{0}^{\ell_{c} / v} d z R(t-z)
$$

for $t>\ell_{c} / v$ and $\hat{p}_{t}(v, t)=p_{s}(v)$ for $0<t \leqslant \ell_{c} / v ; R(t)$ is the probability per time that a particle arrives at a turning point at time $t$ (see Appendix A). Thus, the t-Lagrangian velocity PDF is determined by the sampling of the steady s-Lagrangian PDF $p_{s}(v)$ between turning points. The right-hand side of (26) expresses the probability $p_{s}(v)$ of encountering velocity $v$ at a turning point times the probability that the particle has arrived within an interval of length $\ell_{c} / v$ before the observation time. The arrival time frequency $R(t)$ at a turning point satisfies the Kolmogorov-type equation

$$
R(t)=\delta(t)+\int_{0}^{t} d t^{\prime} R\left(t^{\prime}\right) \psi\left(t-t^{\prime}\right)
$$

The probability per time to just arrive at a turning point is equal to the probability to be at a turning point at any time $t^{\prime}$ times the probability to make a transition of duration $t-t^{\prime}$ to arrive at the next turning point. The t-Lagrangian velocity PDF (26) is nonstationary.

From (27) the Laplace space solution for $R^{*}(\lambda)$ is

$$
R^{*}(\lambda)=\frac{1}{1-\psi^{*}(\lambda)}
$$

In the limit $\lambda \tau_{v} \ll 1$, it can be approximated by $R^{*}(\lambda)=\left(\lambda \tau_{v}\right)^{-1}+\cdots$ and therefore for $t \gg \tau_{v}$ we approximate $R(t)=\tau_{v}^{-1}+\cdots$. Thus, in the limit of $t \gg \tau_{v}$, we obtain from (26),

$$
\hat{p}_{t}(v, t)=p_{e}(v)+\cdots .
$$

Thus, asymptotically, $\hat{p}_{t}(v, t)$ converges toward the Eulerian velocity $\operatorname{PDF} p_{e}(v)$.

Similarly, we obtain for the two-point PDF of the t-Lagrangian velocity the equation

$$
\hat{p}_{t}\left(v, t ; v^{\prime}, t^{\prime}\right)=p_{s}\left(v^{\prime}\right) \int_{0}^{\ell_{c} / v^{\prime}} d z^{\prime} \hat{p}\left(v, t-t^{\prime}+z^{\prime}\right) R\left(t^{\prime}-z^{\prime}\right)
$$


where $t>t^{\prime}$ (see Appendix A). It is nonstationary as indicated by its explicit dependence on $t^{\prime}$. Again, in the limit $t, t^{\prime} \gg \tau_{v}$, we approximate

$$
\hat{p}_{t}\left(v, t ; v^{\prime}, t^{\prime}\right)=p_{e}\left(v^{\prime}\right) \hat{p}\left(v, t-t^{\prime}\right) .
$$

It is therefore asymptotically stationary.

In summary, the classical CTRW describes the evolution of the t-Lagrangian velocity PDF from the flux-weighted Eulerian to the Eulerian velocity PDF. The t-Lagrangian velocities are nonstationary [28]. This property is also called aging in the literature [21]. In the following, we analyze a CTRW formulation that allows for stationary t-Lagrangian statistics and accounts for the evolutions of the t- and s-Lagrangian velocity PDFs from any initial distribution.

\section{B. Markov process of s-Lagrangian velocities}

In order to introduce correlations between subsequent particle velocities and thus quantify the evolution of Lagrangian velocity statistics, we describe the velocity series $v_{s}(s)$ measured equidistantly along a streamline as a Markov process $[2,13,15,29]$. The evolution of the s-Lagrangian velocity PDF is now given by the Chapman-Kolmogorov equation

$$
\hat{p}_{s}(v, s+\Delta s)=\int_{0}^{\infty} d v^{\prime} r\left(v, \Delta s \mid v^{\prime}\right) \hat{p}_{s}\left(v^{\prime}, s\right)
$$

where we assume that the transition $\operatorname{PDF} r\left(v, s \mid v, s^{\prime}\right) \equiv r\left(v, s-s^{\prime} \mid v^{\prime}\right)$ is stationary in $s$. The evolution of particle time in this CTRW is given by

$$
t(s+\Delta s)=t(s)+\frac{\Delta s}{v_{s}(s)} .
$$

The joint Markov process $\left[v_{s}(s), t(s)\right]$ of streamwise velocity and time is characterized by the joint transition density

$$
\psi\left(v, t-t^{\prime}, \Delta s \mid v^{\prime}\right)=r\left(v, \Delta s \mid v^{\prime}\right) \delta\left(t-t^{\prime}-\Delta s / v^{\prime}\right) .
$$

Note that a Markov chain may be characterized by the convergence rate of the transition PDF $r\left(v, n \Delta s \mid v^{\prime}\right)$ toward its steady state, which here is given by

$$
\lim _{n \rightarrow \infty} r\left(v, n \Delta s \mid v^{\prime}\right)=p_{s}(v) .
$$

The (spatial) convergence rate is given by the inverse of the correlation distance $\ell_{c}$ along the streamline. We consider now a process that is uniquely characterized by the steady-state PDF $p_{s}(v)$ and the streamwise correlation distance $\ell_{c}$ and model the s-Lagrangian velocity series by the stochastic relaxation process

$$
v_{s}(s+\Delta s)=[1-\xi(s)] v(s)+\xi(s) v(s) .
$$

The random velocities $v(s)$ are identical and independently distributed according to the steady s-Lagrangian velocity PDF $p_{s}(v)$. The $\xi(s)$ are identical and independently distributed Bernoulli variables that take the value 1 with probability $1-\exp \left(-\Delta s / \ell_{c}\right)$ and 0 with probability $\exp \left(-\Delta s / \ell_{c}\right)$. Thus, its PDF is

$$
p_{\xi}(\xi)=\exp \left(-\Delta s / \ell_{c}\right) \delta(\xi)+\left[1-\exp \left(-\Delta s / \ell_{c}\right)\right] \delta(\xi-1)
$$

The initial velocity distribution is given by $p_{0}(v)$. The transition probability $r\left(v, s \mid v^{\prime}\right)$ for the process (33d) is given by

$$
r\left(v, s \mid v^{\prime}\right)=\exp \left(-s / \ell_{c}\right) \delta\left(v-v^{\prime}\right)+\left[1-\exp \left(-s / \ell_{c}\right)\right] p_{s}(v) .
$$

The velocity process is fully defined by the transition PDF (33f) and the PDF $p_{0}(v)$ of initial velocities. 


\section{Space-Lagrangian velocity statistics}

Using the explicit expression (33f) in Eq. (32) and performing the continuum limit $\Delta s \rightarrow 0$, we obtain the following master equation for the streamwise evolution of $\hat{p}_{s}(v, s)$ :

$$
\frac{\partial \hat{p}_{s}(v, s)}{\partial s}=\ell_{c}^{-1}\left[p_{s}(v)-\hat{p}_{s}(v, s)\right],
$$

subject to the initial condition $\hat{p}_{s}(v, s=0)=p_{0}(v)$. Its solution

$$
\hat{p}_{s}(v, s)=p_{s}(v)+\exp \left(-s / \ell_{c}\right)\left[p_{0}(v)-p_{s}(v)\right]
$$

converges exponentially from $p_{0}(v)$ toward the steady-state distribution $p_{s}(v)$ and for $p_{0}(v)=p_{s}(v)$ it is stationary. The mean s-Lagrangian velocity is defined by

$$
\left\langle v_{s}(s)\right\rangle=\int_{0}^{\infty} d v v \hat{p}_{s}(v, s)
$$

and from (35) we obtain the explicit expression

$$
\left\langle v_{s}(s)\right\rangle=\left\langle v_{s}\right\rangle+\exp \left(-s / \ell_{c}\right)\left[\left\langle v_{0}\right\rangle-\left\langle v_{s}\right\rangle\right] .
$$

Under stationary conditions, this means, for $v_{0}=v_{s}$, it is constant equal to $\left\langle v_{s}\right\rangle$.

The velocity covariance is then defined by

$$
C_{s}\left(s, s^{\prime}\right)=\left\langle v_{s}(s) v_{s}\left(s^{\prime}\right)\right\rangle-\left\langle v_{s}(s)\right\rangle\left\langle v_{s}\left(s^{\prime}\right)\right\rangle,
$$

where the velocity cross moment is

$$
\left\langle v_{s}(s) v_{s}\left(s^{\prime}\right)\right\rangle=\int_{0}^{\infty} d v \int_{0}^{\infty} d v^{\prime} v v^{\prime} r\left(v, s-s^{\prime} \mid v^{\prime}\right) p_{s}\left(v^{\prime}, s^{\prime}\right)
$$

for $s>s^{\prime}$. Using (34) and (33f), we obtain for $s>s^{\prime}$, the explicit expression

$$
\begin{aligned}
C_{s}\left(s, s^{\prime}\right)= & \left(\left\langle v_{0}\right\rangle-\left\langle v_{s}\right\rangle\right)^{2} \exp \left(-s / \ell_{c}\right)\left[1-\exp \left(-s^{\prime} / \ell_{c}\right)\right]+\sigma_{v_{s}}^{2} \exp \left[-\left(s-s^{\prime}\right) / \ell_{c}\right] \\
& +\left(\sigma_{v_{0}}^{2}-\sigma_{v_{s}}^{2}\right) \exp \left(-s / \ell_{c}\right) .
\end{aligned}
$$

For stationary initial velocities $v_{0}=v_{s}$, it reduces to $C_{s}\left(s, s^{\prime}\right) \equiv C_{s}\left(s-s^{\prime}\right)=\sigma_{v_{s}}^{2} \exp \left[-\left(s-s^{\prime}\right) / \ell_{c}\right]$.

\section{Time-Lagrangian velocity statistics}

Here we quantify the temporal evolution of the Lagrangian velocity distribution. The existence of a spatial correlation length entails short-range correlation in space and long-range correlation in time for the Lagrangian velocities, which we quantify in the following.

In the continuum limit of $\Delta s \rightarrow 0$, the time process (33a) becomes

$$
\frac{d t(s)}{d s}=\frac{1}{v_{s}(s)} .
$$

The conjugate process $s(t)$, which is the distance traveled along the streamline until time $t$, is defined by $s(t)=\sup \{s \mid t(s) \leqslant t\}$. The t-Lagrangian velocities $v_{t}(t)$ are now given in terms of $v_{s}(s)$ as

$$
v_{t}(t)=v_{s}[s(t)]
$$

a. One-point statistics. Thus, the t-Lagrangian velocity PDF reads now

$$
\hat{p}_{t}(v, t)=\left\langle\delta\left(v-v_{s}[s(t)]\right)\right\rangle .
$$

Using the properties of the Dirac $\delta$, we can expand this equation into

$$
\hat{p}(v, t)=\int_{0}^{\infty} d s v^{-1} R(v, t, s)
$$


where we defined the probability density $R(v, t, s)$ that a particle has the velocity $v$ and the time $t$ at a distance $s$ along the trajectory as

$$
R(v, t, s)=\langle\delta[v-v(s)] \delta[t-t(s)]\rangle .
$$

Note that $R(v, t, s)$ is the density of the joint Markov process (33) for [ $\left.v_{s}(s), t(s)\right]$. Thus, it satisfies the Chapman-Kolmogorov equation

$$
R(v, t, s+\Delta s)=\int_{0}^{\infty} d v^{\prime} \int_{0}^{t} d z \psi\left(v, t-z, \Delta s \mid v^{\prime}\right) R\left(v^{\prime}, z, s\right) .
$$

Inserting (33b) and (33f) into the right-hand side of (46) and taking the limit $\Delta s \rightarrow 0$ gives the master equation (see Appendix B)

$$
\frac{\partial R(v, t, s)}{\partial s}=-\ell_{c}^{-1} R(v, t, s)-v^{-1} \frac{\partial R(v, t, s)}{\partial t}+\ell_{c}^{-1} p_{s}(v) \int_{0}^{\infty} d v^{\prime} R\left(v^{\prime}, t, s\right),
$$

with the initial condition $R(v, t, s=0)=p_{0}(v) \delta(t)$. Integrating this equation over $s$ according to (44) gives for the t-Lagrangian velocity PDF the integro-differential equation

$$
\frac{\partial \hat{p}_{t}(v, t)}{\partial t}=-\frac{v}{\ell_{c}} \hat{p}_{t}(v, t)+p_{s}(v) \int_{0}^{\infty} d v^{\prime} \frac{v^{\prime}}{\ell_{c}} \hat{p}_{t}\left(v^{\prime}, t\right),
$$

with the initial condition $\hat{p}(v, t=0)=p_{0}(v)$. Its solution in Laplace space is given by (see Appendix B)

$$
\hat{p}_{t}^{*}(v, \lambda)=p_{0}(v) g_{0}^{*}(v, \lambda)+\frac{v}{\left\langle v_{e}\right\rangle} \frac{p_{e}(v) g_{0}^{*}(v, \lambda) \psi_{0}^{*}(\lambda)}{1-\psi_{s}^{*}(\lambda)},
$$

where we defined the propagator

$$
g_{0}(v, t)=\exp \left(-t v / \ell_{c}\right)
$$

whose Laplace transform is given by $g_{0}^{*}(v, \lambda)=\left(\lambda+v / \ell_{c}\right)^{-1}$. We define the transit time distributions $\psi_{0}(t), \psi_{s}(t)$, and $\psi_{e}(t)$ through

$$
\psi_{i}(t)=\tau_{v}^{-1} \int_{0}^{\infty} d v g_{0}(v, t) \frac{v p_{i}(v)}{\left\langle v_{e}\right\rangle},
$$

with $i=0, s, e$. Note that its initial value is $\psi_{i}(t=0)=\left\langle v_{i}\right\rangle / \ell_{s}$. Its Laplace transform is given by

$$
\psi_{i}^{*}(\lambda)=\tau_{v}^{-1} \int_{0}^{\infty} d v \frac{v p_{i}(v)}{\left(\lambda+v / \ell_{c}\right)\left\langle v_{e}\right\rangle} .
$$

It can be seen from (49) that $\hat{p}(v, t)$ is steady for the initial condition $p_{0}(v)=p_{e}(v)$ and is unsteady for any other initial condition by noting that $1-\psi_{s}^{*}(\lambda)=\lambda \tau_{v} \psi_{e}^{*}(\lambda)$.

Expression (49) quantifies the evolution of the t-Lagrangian velocity distribution through potentially long-range temporal correlations reflected by the transit time distributions (51). Note that the transition time PDFs (51) are different from definition (23) for the classical $s$-discrete CTRW framework discussed in Sec. III A.

b. Two-point statistics. The two-point velocity density is defined here by

$$
\hat{p}\left(v, t ; v^{\prime}, t^{\prime}\right)=\left\langle\delta(v-v[s(t)]) \delta\left(v^{\prime}-v\left[s\left(t^{\prime}\right)\right]\right)\right\rangle .
$$

Along the same lines as above, we derive, by using the properties of the Dirac $\delta$,

$$
\hat{p}\left(v, t ; v^{\prime}, t^{\prime}\right)=\int_{0}^{\infty} d s \int_{0}^{\infty} d s^{\prime} v^{-1} R\left(v, t-t^{\prime}, s-s^{\prime} \mid v^{\prime}\right) v^{-1} R\left(v^{\prime}, t^{\prime}, s^{\prime}\right) .
$$

The conditional PDF $R\left(v, t-t^{\prime}, s-s^{\prime} \mid v^{\prime}\right)$ describes the joint distribution of $\left[v_{s}(s), t(s)\right]$ conditional to $v_{s}\left(s^{\prime}\right)=v^{\prime}$ and $t\left(s^{\prime}\right)=t^{\prime}$. It satisfies the master equation (47) with the initial condition 
$R\left(v, t, s=0 \mid v^{\prime}\right)=\delta\left(v-v^{\prime}\right) \delta(t)$. Note that $R\left(v, t-t^{\prime}, s-s^{\prime} \mid v^{\prime}\right)$ is stationary in $t$ and $s$ due to the stationarity of the velocity and time processes as expressed by the transition PDF (33b). Using definition (44), we can now write (54) as

$$
\hat{p}\left(v, t ; v^{\prime}, t^{\prime}\right)=\hat{p}\left(v, t-t^{\prime} \mid v^{\prime}\right) \hat{p}_{t}\left(v^{\prime}, t^{\prime}\right),
$$

where we defined

$$
\hat{p}\left(v, t \mid v^{\prime}\right)=v^{-1} \int_{0}^{\infty} d s R\left(v, t, s \mid v^{\prime}\right)
$$

It satisfies the integro-differential equation (48) for the initial condition $\hat{p}\left(v, t=0 \mid v^{\prime}\right)=\delta\left(v-v^{\prime}\right)$. Its Laplace space solution is obtained from (49) by setting $p_{0}(v)=\delta\left(v-v^{\prime}\right)$ as

$$
\hat{p}_{t}^{*}\left(v, \lambda \mid v^{\prime}\right)=g_{0}^{*}(v, \lambda) \delta\left(v-v^{\prime}\right)+\frac{v v^{\prime}}{\left\langle v_{e}\right\rangle^{2} \tau_{c}} \frac{p_{e}(v) g_{0}^{*}(v, \lambda) g_{0}^{*}\left(v^{\prime}, \lambda\right)}{1-\psi_{s}^{*}(\lambda)},
$$

where we note that here $\psi_{0}^{*}(\lambda)=g_{0}^{*}\left(v^{\prime}, \lambda\right) v^{\prime} / \ell_{c}$. Recall that the one-point PDF $\hat{p}(v, t)$ is stationary and equal to $p_{e}(v)$ for the initial condition $p_{0}(v)=p_{e}(v)$. Under these conditions, the two-point density (55) is

$$
\hat{p}\left(v, t ; v^{\prime}, t^{\prime}\right) \equiv \hat{p}\left(v, t-t^{\prime}, v^{\prime}\right)=\hat{p}\left(v, t-t^{\prime} \mid v^{\prime}\right) p_{e}\left(v^{\prime}\right)
$$

and so is stationary. In the following, we determine the mean and covariance of the t-Lagrangian velocities as well as the corresponding particle dispersion.

\section{VELOCITY MEAN, COVARIANCE, AND DISPERSION}

We study here the t-Lagrangian mean velocity, its covariance, and the particle dispersion for the CTRW model presented in Sec. III B. We investigate these quantities for the $\Gamma$ distribution of Eulerian velocities

$$
p_{e}(v)=\frac{\left(v / v_{0}\right)^{\alpha-1} \exp \left(-v / v_{0}\right)}{v_{0} \Gamma(\alpha)}
$$

for $\alpha>0$, which provides a parametric model for the low end of Eulerian velocity distributions in porous media both on the pore and on the Darcy scale [5,9]. As initial conditions we consider either the Eulerian (59) or steady s-Lagrangian velocity PDF (10), which is obtained from the Eulerian velocity PDF through flux weighting

$$
p_{s}(v)=\frac{\left(v / v_{0}\right)^{\alpha} \exp \left(-v / v_{0}\right)}{v_{0} \Gamma(\alpha+1)} .
$$

Note that the Eulerian and flux-weighted mean and mean-square velocities are

$$
\begin{gathered}
\left\langle v_{e}\right\rangle=v_{0} \alpha \quad\left\langle v_{e}^{2}\right\rangle=v_{0}^{2} \alpha(\alpha+1), \\
\left\langle v_{s}\right\rangle=v_{0}(\alpha+1), \quad\left\langle v_{s}^{2}\right\rangle=v_{0}^{2}(\alpha+1)(\alpha+2) .
\end{gathered}
$$

Inserting (59) into (51), we obtain for the transit time distribution $\psi_{e}(t)$,

$$
\psi_{e}(t)=\frac{\alpha}{\tau_{0}\left(1+t / \tau_{0}\right)^{1+\alpha}},
$$

where $\tau_{0}=\ell_{c} / v_{0}$. For the transit time distribution $\psi_{s}(t)$, we obtain analogously,

$$
\psi_{s}(t)=\frac{\alpha+1}{\tau_{0}\left(1+t / \tau_{0}\right)^{2+\alpha}} .
$$


The Laplace transforms of $\psi_{e}(t)$ and $\psi_{s}(t)$ can be expanded by using Tauberian theorems. For $0<\alpha<1, \psi_{e}^{*}(\lambda)$ is

$$
\psi_{e}^{*}(\lambda)=1-a_{\alpha}\left(\lambda \tau_{0}\right)^{\alpha}
$$

where $a_{\alpha}=\Gamma(1-\alpha)$. For $\alpha=1$ we have

$$
\psi_{e}^{*}(\lambda)=1+\lambda \tau_{0} \ln \left(\lambda \tau_{0}\right)
$$

In the range $0<\alpha<1$, we obtain for $\psi_{s}^{*}(\lambda)$ the expansion

$$
\psi_{s}^{*}(\lambda)=1-\lambda \tau_{v}+b_{\alpha}\left(\lambda \tau_{0}\right)^{1+\alpha},
$$

where $\tau_{v}=\ell_{c} / \alpha v_{0}$ and $b_{\alpha}=\Gamma(2-\alpha)$. For $\alpha=1$ one obtains

$$
\psi_{s}^{*}(\lambda)=1-\lambda \tau_{0}-\left(\lambda \tau_{0}\right)^{2} \ln \left(\lambda \tau_{0}\right) .
$$

Note that the case $\alpha=1$ corresponds to an exponential distribution of Eulerian velocities. For $\alpha>1$, both the first and second moments of $\psi_{s}(t)$ exist such that $\psi_{s}^{*}(\lambda)$ can be expanded as

$$
\psi_{s}^{*}(\lambda)=1-\lambda \tau_{v}+\frac{\left\langle\tau_{s}^{2}\right\rangle}{2} \lambda^{2}
$$

In the following, we will discuss the mean t-Lagrangian velocity, the velocity covariance, and particle dispersion. We present general Laplace space expressions based on the explicit expressions for the one- and two-point velocity PDFs derived in Sec. III B 2 and study their temporal behavior for the Eulerian velocity PDF given by the $\Gamma$ distribution (59). To this end, we perform random walk particle tracking simulations based on (33) and derive explicit expressions for the early- and late-time behaviors using the expansions (67)-(69) of the Laplace transform of the streamwise transition time $\operatorname{PDF} \psi_{s}(t)$.

\section{A. Mean velocity}

The mean particle velocity is equal to the one-point t-Lagrangian velocity moment

$$
m_{1}(t)=\int_{0}^{\infty} d v v \hat{p}_{t}(v, t) .
$$

Using (49), we obtain for the Laplace transform of $m_{1}(t)$,

$$
m_{1}^{*}(\lambda)=\ell_{c} \psi_{0}^{*}(\lambda)+\int_{0}^{\infty} d v \frac{v^{2}}{\left\langle v_{e}\right\rangle} \frac{p_{e}(v) g_{0}^{*}(v, \lambda) \psi_{0}^{*}(\lambda)}{1-\psi_{s}^{*}(\lambda)}
$$

For the stationary initial conditions $p_{0}(v)=p_{e}(v)$, the particle velocity is constant $m_{1}(t)=\left\langle v_{e}\right\rangle$ and equal to the mean Eulerian velocity.

For the nonstationary initial conditions $p_{0}(v)=p_{s}(v)$ we obtain at short times $t \ll \tau_{v}$,

$$
m_{1}(t)=\ell_{c} \psi_{s}(t)
$$

This means that it decreases from its initial value $\left\langle v_{s}\right\rangle$ as $\psi_{s}(t)$. For times $t \gg \tau_{v}$ and $0<\alpha<1$, we use the expansion (67) in Eq. (71), which gives in leading order

$$
m_{1}^{*}(\lambda)=\frac{\left\langle v_{e}\right\rangle}{\lambda}+\frac{\left\langle v_{e}\right\rangle \tau_{0} b_{\alpha}}{b_{1}}\left(\lambda \tau_{0}\right)^{\alpha-1} .
$$

For $\alpha=1$ we obtain

$$
m_{1}^{*}(\lambda)=\frac{\left\langle v_{e}\right\rangle}{\lambda}-\ell_{c} \ln \left(\lambda \tau_{0}\right)
$$

Thus, the long-time behavior of $m_{1}(t)$ for $0<\alpha \leqslant 1$ is

$$
m_{1}(t)=\left\langle v_{e}\right\rangle+c\left\langle v_{e}\right\rangle\left(t / \tau_{0}\right)^{-\alpha}
$$



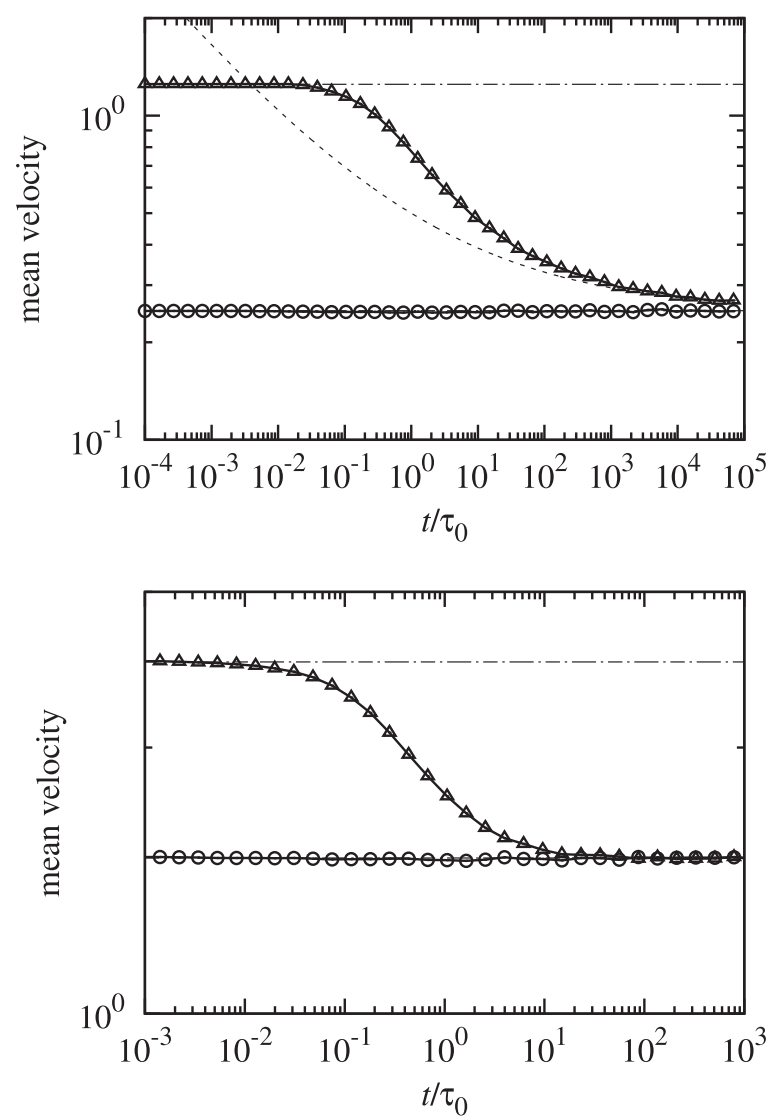

FIG. 1. Evolution of the mean velocity under stationary and nonstationary conditions for $p_{0}(v)=p_{e}(v)$ (circles) and $p_{0}(v)=p_{s}(v)$ (triangles) for $\alpha=1 / 4$ (top) and $\alpha=3 / 2$ (bottom). The dashed line in the top panel indicates the asymptotic behavior (75). The dash-dotted lines indicate the average stationary s-Lagrangian and Eulerian velocities. The numerical random walk simulation that produced these data is based on (33) for $\Delta s=10^{-2} \ell_{c}$ and $10^{5}$ particles.

where we defined $c=b_{\alpha} / \Gamma(1-\alpha) b_{1}$ for $0<\alpha<1$ and $c=1$ for $\alpha=1$. This means that the mean velocity converges as a power law toward its asymptotic value, which is given by the Eulerian mean velocity.

For $\alpha>1$ we use (69) in order to obtain in leading order for $\lambda \ll \tau_{0}$,

$$
m_{1}^{*}(\lambda)=\frac{\left\langle v_{e}\right\rangle}{\lambda}+\ell_{c}+\left\langle v_{e}\right\rangle \frac{\left\langle\tau_{s}^{2}\right\rangle}{2 \tau_{v}}
$$

This means that for $t \gg \tau_{v}, m_{1}(t)$ can be written as

$$
m_{1}(t)=\left\langle v_{e}\right\rangle+\left(\ell_{c}+\left\langle v_{e}\right\rangle \frac{\left\langle\tau_{s}^{2}\right\rangle}{2 \tau_{v}}\right) \delta(t) .
$$

Note that the Dirac $\delta$ indicates that the convergence toward its asymptotic value is faster than $1 / t$. These behaviors are illustrated in Fig. 1, which shows the evolution of the t-Lagrangian mean velocity with time under Eulerian and flux-weighted Eulerian initial conditions for $\alpha=1 / 4$ and $\alpha=3 / 2$. 


\section{B. Velocity covariance}

The t-Lagrangian velocity covariance is given by

$$
C_{t}\left(t, t^{\prime}\right)=m_{2}\left(t, t^{\prime}\right)-m_{1}(t) m_{1}\left(t^{\prime}\right),
$$

where we defined the two-point velocity moment by

$$
m_{2}\left(t, t^{\prime}\right)=\int_{0}^{\infty} d v \int_{0}^{\infty} d v^{\prime} v v^{\prime} \hat{p}_{t}\left(v, t ; v^{\prime}, t^{\prime}\right),
$$

which can be written in terms of (55) for the two-point velocity PDF as

$$
m_{2}\left(t, t^{\prime}\right)=\int_{0}^{\infty} d v^{\prime} m_{1}\left(t-t^{\prime} \mid v^{\prime}\right) v^{\prime} \hat{p}_{t}\left(v^{\prime}, t^{\prime}\right),
$$

where we defined the conditional velocity moment as

$$
m_{1}\left(t \mid v^{\prime}\right)=\int_{0}^{\infty} d v v \hat{p}_{t}\left(v, t \mid v^{\prime}\right)
$$

The Laplace transform of (81) is then obtained from (57) as

$$
m_{1}^{*}\left(\lambda \mid v^{\prime}\right)=v^{\prime} g_{0}^{*}\left(v^{\prime}, \lambda\right)+\int_{0}^{\infty} d v \frac{v^{2} v^{\prime}}{\left\langle v_{e}\right\rangle^{2} \tau_{v}} \frac{p_{e}(v) g_{0}^{*}(v, \lambda) g_{0}^{*}\left(v^{\prime}, \lambda\right)}{1-\psi_{s}^{*}(\lambda)} .
$$

We first consider the case $0<\alpha \leqslant 1$. For times $t \gg \tau_{v}$, which means for $\lambda \tau_{v} \ll 1$, we find by using (67) and (68) in Eq. (82) that the leading order of $m_{1}^{*}\left(\lambda \mid v^{\prime}\right)$ is given by (73) for $0<\alpha<1$ and (74) for $\alpha=1$. Specifically, this implies that $m_{1}\left(t \mid v^{\prime}\right)$ is independent of $v^{\prime}$. Using (75) in Eq. (80), we obtain

$$
m_{2}\left(t, t^{\prime}\right)=\left[\left\langle v_{e}\right\rangle+\frac{c\left\langle v_{e}\right\rangle \tau_{0}^{\alpha}}{\left(t-t^{\prime}\right)^{\alpha}}\right] m_{1}\left(t^{\prime}\right)
$$

Under stationary conditions, $p_{0}(v)=p_{e}(v), m_{1}(t)=\left\langle v_{e}\right\rangle$, and $m_{2}\left(t, t^{\prime}\right) \equiv m_{2}\left(t-t^{\prime}\right)$, hence

$$
m_{2}\left(t-t^{\prime}\right)=\left\langle v_{e}\right\rangle^{2}+\frac{c\left\langle v_{e}\right\rangle^{2} \tau_{0}^{\alpha}}{\left(t-t^{\prime}\right)^{\alpha}} .
$$

Thus, the velocity covariance is stationary and behaves for $\left(t-t^{\prime}\right) \gg \tau_{v}$ and $0<\alpha \leqslant 1$ as

$$
C_{t}\left(t-t^{\prime}\right)=\frac{c\left\langle v_{e}\right\rangle^{2} \tau_{0}^{\alpha}}{\left(t-t^{\prime}\right)^{\alpha}}
$$

This behavior is illustrated in Fig. 2.

Under the nonstationary condition with $p_{0}(v)=p_{s}(v)$, we use the fact that $m_{1}\left(t \mid v^{\prime}\right)=m_{1}(t)$ in the limit $t \gg \tau_{v}$ in order to write

$$
m_{2}\left(t, t^{\prime}\right)=m_{1}\left(t-t^{\prime}\right) m_{1}\left(t^{\prime}\right)
$$

Accordingly, we obtain for the covariance in the limit $\left(t-t^{\prime}\right) \gg \tau_{v}$,

$$
C_{t}\left(t, t^{\prime}\right)=m_{1}\left(t^{\prime}\right)\left[m_{1}\left(t-t^{\prime}\right)-m_{1}(t)\right] .
$$

We now consider the case $\alpha>1$. For $\lambda \tau_{v} \ll 1$, we expand (82) by using (69) to leading order, which gives

$$
m_{1}\left(\lambda \mid v^{\prime}\right)=\frac{\left\langle v_{e}\right\rangle}{\lambda}+\frac{\left\langle v_{e}\right\rangle\left\langle\tau_{s}^{2}\right\rangle}{2 \tau_{v}}-\frac{\left\langle v_{2}\right\rangle \ell_{c}}{v^{\prime}}
$$




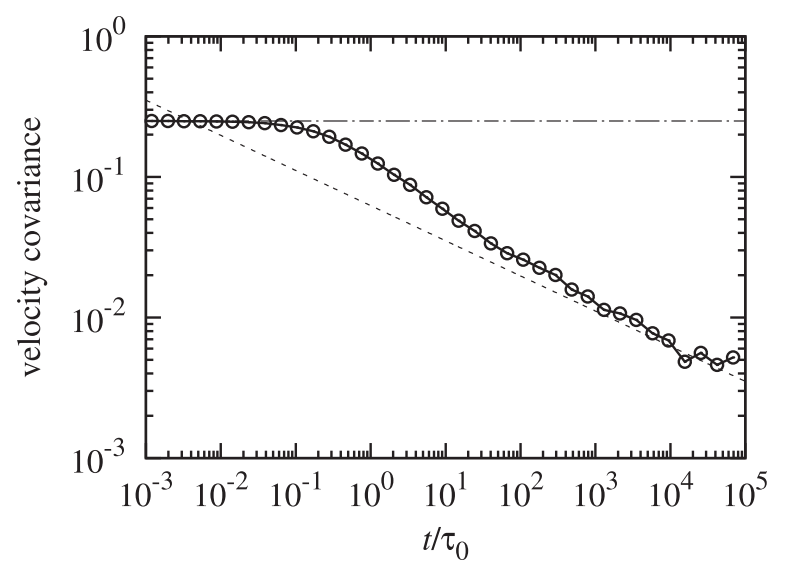

FIG. 2. Covariance of the t-Lagrangian velocity under the stationary condition $p_{0}(v)=p_{e}(v)$ for $\alpha=1 / 4$. The dashed line indicates the asymptotic behavior (85). The dash-dotted line indicates the velocity variance. The numerical random walk simulation that produced these data is based on (33) for $\Delta s=10^{-2} \ell_{c}$ and $10^{5}$ particles.

Thus, we obtain for $m_{2}\left(t, t^{\prime}\right)$,

$$
m_{2}\left(t, t^{\prime}\right)=\left[\left\langle v_{e}\right\rangle+\frac{\left\langle v_{e}\right\rangle\left\langle\tau_{s}^{2}\right\rangle}{2 \tau_{v}} \delta\left(t-t^{\prime}\right)\right] m_{1}\left(t^{\prime}\right)-\ell_{c}\left\langle v_{e}\right\rangle \delta\left(t-t^{\prime}\right) .
$$

For $t-t^{\prime} \gg \tau_{v}$, we obtain for the covariance under both stationary and nonstationary conditions the expression

$$
C_{t}\left(t-t^{\prime}\right)=\ell_{c}\left\langle v_{e}\right\rangle\left(\frac{\left\langle\tau_{s}^{2}\right\rangle}{2 \tau_{v}^{2}}-1\right) \delta\left(t-t^{\prime}\right)
$$

Again note that the Dirac $\delta$ indicates here that the covariance decays faster than $1 / t$. These expressions allow studying the dynamics of dispersion as a function of the Eulerian velocity distribution and the initial injection, as discussed in the following.

\section{Dispersion}

The time-dependent dispersion coefficient $\mathcal{D}(t)$ is obtained from the Green-Kubo relation [30] as the integral of the t-Lagragian velocity correlation as

$$
\mathcal{D}(t)=\int_{0}^{t} d t^{\prime} C_{t}\left(t, t^{\prime}\right)
$$

At time $t \ll \tau_{v}$, subsequent particle velocities are strongly correlated. As a consequence, the dispersion coefficient grows ballistically as

$$
\mathcal{D}(t)=\left\langle\left(v_{0}-\left\langle v_{0}\right\rangle\right)^{2}\right\rangle t .
$$

Thus, for the initial condition $p_{0}(v)=p_{s}(v)$ the ballistic initial growth is faster than for the stationary condition $p_{0}(v)=p_{e}(v)$, because the variance of the flux-weighted $p_{s}(v)$ is larger than the variance of the Eulerian $p_{e}(v)$. For times $t>\tau_{v}$, particle velocities decorrelate from their initial values. High velocities decorrelate faster than low velocities because the characteristic time at which a particle of velocity $v$ makes a velocity transition is given by $\ell_{c} / v$. Thus, at time $\tau_{v}$ most of the particles with $v>\left\langle v_{e}\right\rangle$ have experienced a velocity transition, while particles with $v<\left\langle v_{e}\right\rangle$ persist in their initial velocities. The dispersion coefficient $\mathcal{D}(t)$ then crosses over to its asymptotic long-time behavior, which we study in the following. 

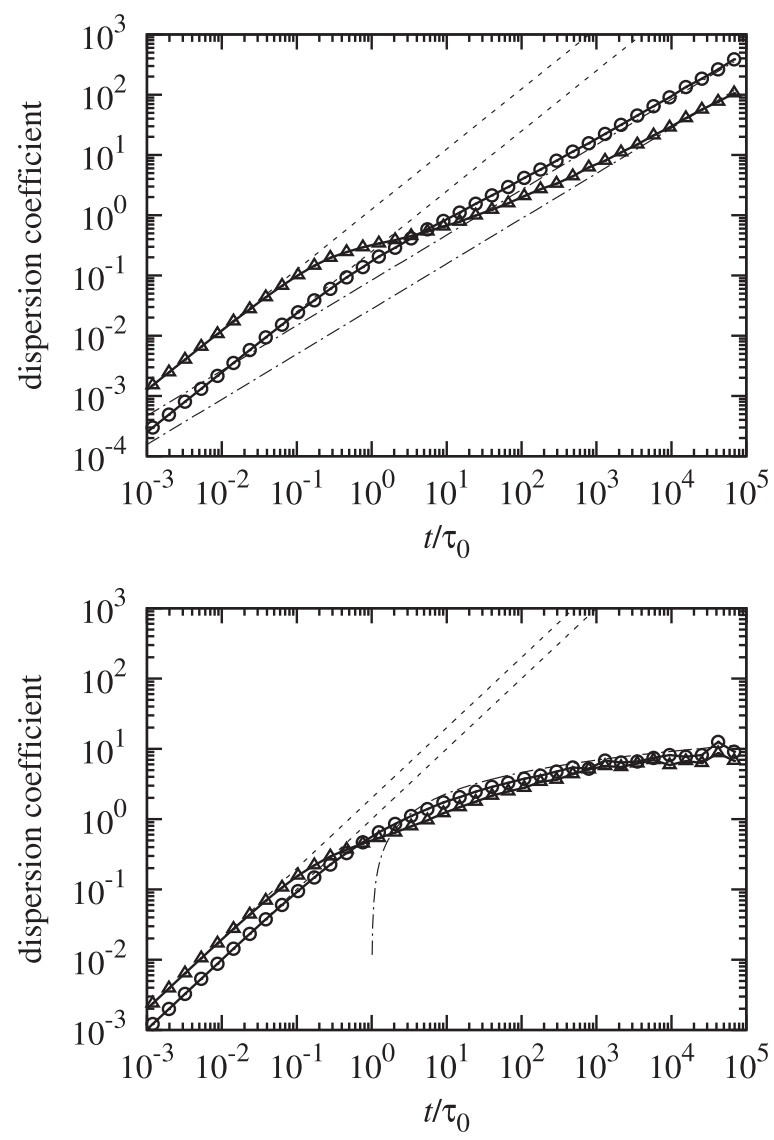

FIG. 3. Evolution of the dispersion coefficient under stationary and nonstationary conditions for $p_{0}(v)=$ $p_{e}(v)$ (circles) and $p_{0}(v)=p_{s}(v)$ (triangles) for $\alpha=1 / 4$ (top) and $\alpha=1$ (bottom). The dashed lines indicate the ballistic behaviors (92) at short times and the dash-dotted lines the asymptotic power-law behaviors (93) and (94) for $\alpha=1 / 4$ and the logarithmic behavior (95) for $\alpha=1$. The numerical random walk simulation that produced these data is based on (33) and $\Delta s=10^{-2} \ell_{c}$ and $10^{5}$ particles.

We first consider the case $0<\alpha \leqslant 1$. Under stationary conditions, which means for $p_{0}(v)=$ $p_{e}(v)$, we obtain from (85), for $t \gg \tau_{v}$ and $0<\alpha<1$,

$$
\mathcal{D}(t)=\left\langle v_{e}\right\rangle \ell_{c} \frac{c \alpha}{1-\alpha}\left(t / \tau_{0}\right)^{1-\alpha} .
$$

Thus, the dispersion behavior is superdiffusive. In the nonstationary case, for $p_{0}(v)=p_{s}(v)$, we obtain from (87) and (75), at $t \gg \tau_{v}$,

$$
\mathcal{D}(t)=\left\langle v_{e}\right\rangle \ell_{c} \frac{c \alpha^{2}}{(1-\alpha)^{2}}\left(t / \tau_{0}\right)^{1-\alpha}
$$

The dispersion coefficient grows asymptotically with the same power law, but slower than in the stationary case. Thus, while the growth rate of particle dispersion is initially larger for the nonstationary initial condition, asymptotically its growth is slower than for the stationary initial velocity PDF. For $\alpha=1$, we obtain for both stationary and nonstationary initial conditions the behavior

$$
\mathcal{D}(t)=\left\langle v_{e}\right\rangle \ell_{c} \ln \left(t / \tau_{0}\right)
$$




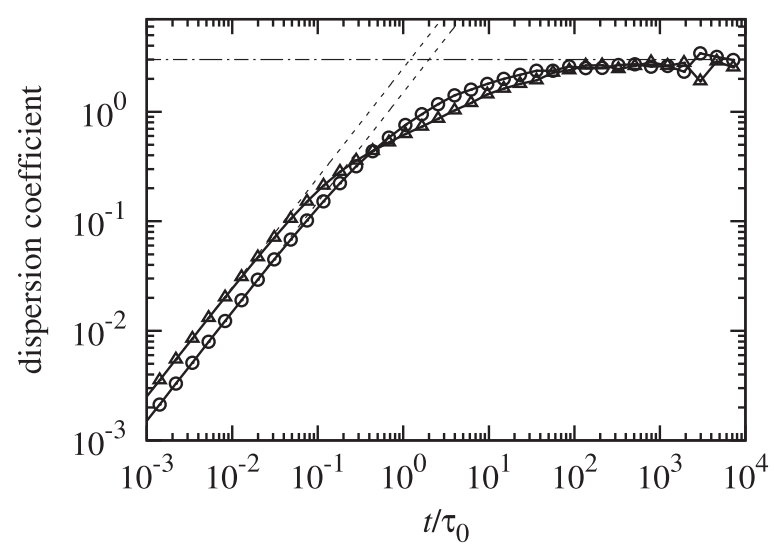

FIG. 4. Evolution of the dispersion coefficient for steady (circles) and unsteady (triangles) initial velocity PDFs for $\alpha=3 / 2$. The dashed lines indicate the ballistic behaviors (92) at short times and the dash-dotted lines the asymptotic long-time value (96). The numerical random walk simulation that produced these data is based on (33) for $\Delta s=10^{-2} \ell_{c}$ and $10^{5}$ particles.

Figure 3 illustrates the evolution of $\mathcal{D}(t)$ for $\alpha=1 / 4$ and $\alpha=1$ under stationary and nonstationary initial conditions. For times $t \ll \tau_{v}$, we observe the ballistic behavior (92), which persists until particle velocities start decorrelating from their initial velocity. Then an intermediate-time regime develops that marks the crossover to the superdiffusive long-time behavior. In this regime, the $\mathcal{D}(t)$ for the nonstationary initial velocity distribution grows slower than for the stationary one. The dispersion behavior here is due to the fluctuations of fast velocities, which have already decorrelated, and low velocity particles that persist in the ballistic mode. The stationary Eulerian initial distribution $p_{e}(v)$ has a stronger weight on low velocities than the flux-weighted $p_{s}(v)$. Thus, dispersion for the former is higher in the intermediate-time regime than for the latter. The end of the intermediate regime is characterized by the decorrelation of most particles from their initial velocities. In the long-time regime, we observe for $0<\alpha<1$ the power-law behaviors (93) and (94), for stationary and nonstationary initial conditions. The difference persists and the dispersion coefficient for stationary initial conditions is larger than for nonstationary. The power-law scalings (93) and (94) are consistent with the ones observed in the CTRW for uncorrelated particle velocities [31,32]. For $\alpha=1$, we observe the logarithmic behavior (95) for both stationary and nonstationary initial conditions.

For $\alpha>1$, the dispersion coefficient converges for $t \gg \tau_{v}$ for both stationary and nonstationary initial conditions toward the constant asymptotic long-time value

$$
\mathcal{D}^{e}=\left\langle v_{e}\right\rangle \ell_{c}\left(\frac{\left\langle\tau_{s}^{2}\right\rangle}{2 \tau_{v}^{2}}-1\right) .
$$

Figure 4 illustrates the evolution of the dispersion coefficient toward the asymptotic value for $\alpha=3 / 2$. At short times $t \ll \tau_{v}$, both dispersion coefficients evolve ballistically; again, the one for the nonstationary initial condition evolves faster. Then, for $t>\tau_{v}$, the dispersion coefficient for stationary initial conditions grows faster than for nonstationary. As pointed out above, the dispersion behavior is due to the fluctuations of decorrelated fast velocities and persistent low velocity. As the stationary Eulerian initial velocity distribution gives a higher probability to low velocities than the flux-weighted distribution, the contrast between particle positions increases faster. The asymptotic regime is reached as the particle velocities fully decorrelate from their initial values. For times $t \gg \tau_{v}$ the dispersion coefficients for both stationary and nonstationary initial conditions converge to the same asymptotic long-time value (96). 


\section{CONCLUSION}

We developed a CTRW approach for the evolution of Lagrangian velocities based on a Markov model for the streamwise equidistant Lagrangian velocities in the form of a stochastic relaxation process. The CTRW framework provides a natural formalism to quantify the impact of the persistence of particle velocities in space on the Lagrangian velocity statistics in time. It has been used to quantify intermittent particle velocities and accelerations for flow through pore- and Darcy-scale porous media, in which flow velocities vary on a characteristic length scale. The velocity statistics in CTRW formulations based on independent successive particle velocities are in general nonstationary. This, however, is not necessarily the case for particle motion through heterogeneous flow fields. Specifically, under Eulerian and Lagrangian ergodicity, the stationarity of the Lagrangian velocity series depends on the initial velocity distribution.

In order to shed light on these dynamics, we first discussed the relation between the Eulerian flow properties and the t-Lagrangian and s-Lagrangian velocities. The t-Lagrangian velocities are defined as the particle velocities sampled isochrone along a streamline, the s-Lagrangian velocities accordingly through equidistant sampling. We found that the PDFs of s- and t-Lagrangian velocities are related through flux weighting. This can be understood from the fact that isochrone sampling gives a higher weight to low velocities because particles spend more time at low velocities, while equidistant sampling assigns the same weight to high and low velocities. Under Eulerian and Lagrangian ergodicity and for volume conserving flows, the Eulerian velocity PDF is equal to the t-Lagrangian PDF. This gives a direct relation between the s-Lagrangian velocity PDF, a transport property, and the Eulerian PDF, a flow property, via flux weighting. We then showed that t-Lagrangian velocities are stationary if their initial distribution is equal to the Eulerian one, while s-Lagrangian velocities are stationary if their initial distribution is given by the flux-weighted Eulerian distribution.

Based on these considerations, we analyzed the t-Lagrangian velocity statistics in the $s$-discrete CTRW characterized by independent velocities with a unique velocity distribution. In classical CTRW approaches, the velocity statistics are in general nonstationary. Thus, we introduced a CTRW that is defined through a Markovian velocity process, for which we use a stochastic relaxation relation that is characterized by the steady-state s-Lagrangian velocity PDF and the correlation length along the streamlines. Based on this, we defined a CTRW approach that models the evolution of Lagrangian velocities from arbitrary initial conditions and yields stationary and nonstationary $\mathrm{s}$ - and t-Lagrangian velocity series. Specifically, this CTRW is $s$ continuous, which means that the streamwise s-Lagrangian velocity is defined at any point along the streamline and its distribution evolves continuously in $s$. We determined the evolution equations and solutions for the Lagrangian one- and two-point statistics and discussed the evolution of the mean particle velocity, covariance, and dispersion under stationary and nonstationary initial conditions. We applied these results to a $\Gamma$ distribution of Eulerian velocities, which serves as a model for heavy-tailed flow statistics through porous media. The low end of the velocity spectrum here scales as $p_{e}(v) \propto v^{\alpha-1}$. For $0<\alpha \leqslant 1$ we found strong velocity correlations and anomalous dispersion, which means here a power-law or logarithmic evolution of the dispersion coefficient with time, while for $\alpha>1$ it evolves toward a constant. These behaviors are fully determined by the Eulerian velocity PDF and the streamwise correlation length. The asymptotic scalings for dispersion are similar as the ones obtained in a corresponding discrete CTRW, as they are attained when particle velocities decorrelate. Their evolution, however, depends on the initial velocity distributions and can be quite different under stationary and nonstationary conditions.

The developed approach sheds light on the modeling and understanding of Lagrangian velocity series in heterogeneous flows and their evolution under stationary and nonstationary conditions. It provides a bridge between CTRW based modeling approaches of particle transport and stochastic transport approaches that start from the representation of the Eulerian velocity field or the medium structure as spatial random fields. The developed CTRW is fully characterized in terms of the Eulerian velocity PDF and the streamwise correlation length, which allows us to predict Lagrangian particle dynamics based on the flow or medium properties. 


\section{ACKNOWLEDGMENT}

M.D. and A.C. acknowledge support from the European Research Council through Project MHetScale (No. 617511).

\section{APPENDIX A: VELOCITY STATISTICS FOR UNCORRELATED S-LAGRANGIAN VELOCITIES}

The one-point t-Lagrangian velocity PDF (25) can be expanded as

$$
\hat{p}(v, t)=\int_{0}^{t} d t^{\prime} \sum_{n=0}^{\infty}\left\langle\delta\left(v-v_{n}\right) \delta\left(t^{\prime}-t_{n}\right) \delta_{n, n_{t}}\right\rangle,
$$

where $\delta_{i j}$ denotes the Kronecker delta. Note that $\delta_{n, n_{t}} \equiv \mathbb{I}\left(t_{n} \leqslant t<t_{n+1}\right)$. Thus, we can write (A1) as

$$
\hat{p}(v, t)=\int_{0}^{t} d t^{\prime} \sum_{n=0}^{\infty}\left\langle\delta\left(v-v_{n}\right) \delta\left(t^{\prime}-t_{n}\right)\right\rangle \mathbb{I}\left(0 \leqslant t-t^{\prime}<\ell_{c} / v\right),
$$

where we have used that $t_{n}$ is independent of $v_{n}$ and that, per the Dirac $\delta$, the $v_{n}$ in the indicator function is set equal to $v$. We further obtain

$$
\hat{p}(v, t)=\int_{t-\ell_{c} / v}^{t} d t^{\prime} \sum_{n=0}^{\infty}\left\langle\delta\left(v-v_{n}\right)\right\rangle\left\langle\delta\left(t^{\prime}-t_{n}\right)\right\rangle \equiv p_{s}(v) \int_{t-\ell_{c} / v}^{t} d t^{\prime} \sum_{n=0}^{\infty} R_{n}\left(t^{\prime}\right)
$$

for $t>\ell_{c} / v ; R_{n}(t)$ is the PDF of $t_{n}$. As $t_{n}$ is a Markov process in step number, we have the Chapman-Kolmogorov equation for the conditional PDF $R_{n, n^{\prime}}\left(t \mid t^{\prime}\right)$,

$$
R_{n+1, n^{\prime}}\left(t \mid t^{\prime}\right)=\int_{t^{\prime}}^{t} d z \psi(t-z) R_{n, n^{\prime}}\left(z \mid t^{\prime}\right)
$$

As the process is homogeneous in $n$ and in $t$, we have that $R_{n^{\prime}+m, n^{\prime}}\left(t \mid t^{\prime}\right) \equiv R_{m}\left(t-t^{\prime}\right)$. The sum over $R_{n}(t)$,

$$
R(t)=\sum_{n=0}^{\infty} R_{n}\left(t^{\prime}\right)
$$

satisfies the integral equation (27).

For the two-point PDF, we obtain in analogy to (A3),

$$
\hat{p}\left(v, t ; v^{\prime}, t^{\prime}\right)=p_{s}(v) p_{s}\left(v^{\prime}\right) \int_{t-\ell_{c} / v}^{t} d z \int_{t^{\prime}-\ell_{c} / v^{\prime}}^{t^{\prime}} d z^{\prime} \sum_{n=0}^{\infty} \sum_{n^{\prime}=0}^{\infty} R_{n, n^{\prime}}\left(z, z^{\prime}\right),
$$

where $R_{n, n^{\prime}}\left(z, z^{\prime}\right)$ is the joint density of $t_{n}$ and $t_{n^{\prime}}$, which can be written as

$$
R_{n^{\prime}+m, n^{\prime}}\left(z, z^{\prime}\right)=R_{m}\left(z-z^{\prime}\right) R_{n^{\prime}}\left(z^{\prime}\right) .
$$

We used the stationarity of the conditional PDF discussed above. Thus, we obtain now

$$
\begin{aligned}
\hat{p}\left(v, t ; v^{\prime}, t^{\prime}\right) & =p_{s}(v) p_{s}\left(v^{\prime}\right) \int_{t-\ell_{c} / v}^{t} d z \int_{t^{\prime}-\ell_{c} / v^{\prime}}^{t^{\prime}} d z^{\prime} \sum_{m=0}^{\infty} \sum_{n^{\prime}=0}^{\infty} R_{m}\left(z-z^{\prime}\right) R_{n^{\prime}}\left(z^{\prime}\right) \\
& \equiv p_{s}(v) p_{s}\left(v^{\prime}\right) \int_{t-\ell_{c} / v}^{t} d z \int_{t^{\prime}-\ell_{c} / v^{\prime}}^{t^{\prime}} d z^{\prime} R\left(z-z^{\prime}\right) R\left(z^{\prime}\right) .
\end{aligned}
$$


Shifting $z \rightarrow t-z$ and $z^{\prime} \rightarrow t^{\prime}-z^{\prime}$ gives

$$
\hat{p}\left(v, t ; v^{\prime}, t^{\prime}\right)=p_{s}(v) p_{s}\left(v^{\prime}\right) \int_{0}^{\ell_{c} / v} d z \int_{0}^{\ell_{c} / v^{\prime}} d z^{\prime} R\left(t-t^{\prime}+z^{\prime}-z\right) R\left(t^{\prime}-z^{\prime}\right) .
$$

Using now expression (26) gives (30).

\section{APPENDIX B: VELOCITY STATISTICS FOR MARKOV PROCESS OF S-LAGRANGIAN VELOCITIES}

The master equation (47) for $R(v, t, s)$ follows from the Chapman-Kolmogorov equation (46) in the limit $\Delta s \rightarrow 0$. In fact, inserting (33b) and (33f) gives

$$
\begin{aligned}
R(v, t, s+\Delta s)= & \exp \left(-\Delta s / \ell_{c}\right) R(v, t-\Delta s / v, s) \\
& +\left[1-\exp \left(-\Delta s / \ell_{c}\right)\right] p_{s}(v) \int_{0}^{\infty} d v^{\prime} R\left(v^{\prime}, t-\Delta s / v^{\prime}, s\right) .
\end{aligned}
$$

Expanding the left and right sides for small $\Delta s$ gives

$$
\begin{aligned}
& R(v, t, s)+\Delta s \frac{\partial R(v, t, s)}{\partial s}+\cdots \\
& \quad=R(v, t, s)-\frac{\Delta s}{v} \frac{\partial R(v, t, s)}{\partial t}-\frac{\Delta s}{\ell_{c}} R(v, t, s)+\frac{\Delta s}{\ell_{c}} p_{s}(v) \int_{0}^{\infty} d v^{\prime} R\left(v^{\prime}, t, s\right)+\cdots,
\end{aligned}
$$

where the dots denote higher-order contributions in $\Delta s$. Dividing by $\Delta s$ and taking the limit $\Delta s \rightarrow 0$ gives (47).

We now derive the solution of Eq. (48). To this end, we perform the Laplace transform, which gives

$$
\lambda \hat{p}_{t}^{*}(v, \lambda)=-\frac{v}{\ell_{c}} \hat{p}_{t}^{*}(v, \lambda)+p_{s}(v) \int_{0}^{\infty} d v^{\prime} \frac{v^{\prime}}{\ell_{c}} \hat{p}_{t}^{*}\left(v^{\prime}, \lambda\right) .
$$

This is a Fredholm equation of the second kind with degenerate kernel [33]. It can be written as

$$
\hat{p}_{t}^{*}(v, \lambda)=g_{0}^{*}(v, \lambda) p_{0}(v)+g_{0}^{*}(v, \lambda) p_{s}(v) \int_{0}^{\infty} d v^{\prime} \frac{v^{\prime}}{\ell_{c}} \hat{p}^{*}\left(v^{\prime}, \lambda\right),
$$

where we defined

$$
g_{0}^{*}(v, \lambda)=\frac{1}{\lambda+v / \ell_{c}}
$$

The solution of (B4) has the form

$$
\hat{p}_{t}^{*}(v, \lambda)=g_{0}^{*}(v, \lambda)\left[p_{0}(v)+p_{s}(v) A(v, \lambda)\right] .
$$

Inserting the latter into (B4) gives for $A(v, \lambda)$,

$$
A(v, \lambda)=\frac{\psi_{0}^{*}(\lambda)}{1-\psi_{s}^{*}(\lambda)}
$$

where we defined

$$
\psi_{i}^{*}(\lambda)=\int_{0}^{\infty} d v^{\prime} g_{0}^{*}\left(v^{\prime}, \lambda\right) \frac{v^{\prime}}{\ell_{c}} p_{i}(v)
$$

with $i=0, s$. Inserting (B7) into (B6) and setting $p_{s}(v)=v p_{e}(v) /\left\langle v_{e}\right\rangle$ gives (49). 
[1] S. B. Pope, Turbulent Flows (Cambridge University Press, Cambridge, 2000).

[2] D. W. Meyer and F. Saggini, Testing the Markov hypothesis in fluid flows, Phys. Rev. E 93, 053103 (2016).

[3] P. de Anna, T. Le Borgne, M. Dentz, A. M. Tartakovsky, D. Bolster, and P. Davy, Flow Intermittency, Dispersion, and Correlated Continuous Time Random Walks in Porous Media, Phys. Rev. Lett. 110, 184502 (2013).

[4] P. K. Kang, P. de Anna, J. P. Nunes, B. Bijeljic, M. J. Blunt, and R. Juanes, Pore-scale intermittent velocity structure underpinning anomalous transport through 3-D porous media, Geophys. Res. Lett. 41, 6184 (2014).

[5] M. Holzner, V. L. Morales, M. Willmann, and M. Dentz, Intermittent lagrangian velocities and accelerations in three-dimensional porous medium flow, Phys. Rev. E 92, 013015 (2015).

[6] E. W. Montroll and G. H. Weiss, Random walks on lattices. II. J. Math. Phys. 6, 167 (1965).

[7] H. Scher and M. Lax, Stochastic transport in a disordered solid. I. Theory, Phys. Rev. B 7, 4491 (1973).

[8] R. Metzler and J. Klafter, The random walk's guide to anomalous diffusion: A fractional dynamics approach, Phys. Rep. 339, 1 (2000).

[9] B. Berkowitz, A. Cortis, M Dentz, and H. Scher, Modeling non-Fickian transport in geological formations as a continuous time random walk, Rev. Geophys. 44, RG2003 (2006).

[10] B. Berkowitz and H. Scher, Anomalous Transport in Random Fracture Networks, Phys. Rev. Lett. 79, 4038 (1997).

[11] R. Benke and S. Painter, Modeling conservative tracer transport in fracture networks with a hybrid approach based on the Boltzmann transport equation, Water Resour. Res. 39, 1324 (2003).

[12] S. Painter and V. Cvetkovic, Upscaling discrete fracture network simulations: An alternative to continuum transport models, Water Resour. Res. 41, W02002 (2005).

[13] T. Le Borgne, M. Dentz, and J. Carrera, Spatial Markov processes for modeling Lagrangian particle dynamics in heterogeneous porous media, Phys. Rev. E 78, 026308 (2008).

[14] T. Le Borgne, M. Dentz, and J. Carrera, A Lagrangian Statistical Model for Transport in Highly Heterogeneous Velocity Fields, Phys. Rev. Lett. 101, 090601 (2008).

[15] P. K. Kang, M. Dentz, T. Le Borgne, and R. Juanes, Spatial Markov Model of Anomalous Transport Through Random Lattice Networks, Phys. Rev. Lett. 107, 180602 (2011).

[16] B. Bijeljic, P. Mostaghimi, and M. J. Blunt, Signature of Non-Fickian Solute Transport in Complex Heterogeneous Porous Media, Phys. Rev. Lett. 107, 204502 (2011).

[17] Y. Edery, A. Guadagnini, H. Scher, and B. Berkowitz, Origins of anomalous transport in heterogeneous media: Structural and dynamic control, Water Resour. Res. 50, 1490 (2014).

[18] P. K. Kang, S. Brown, and R. Juanes, Emergence of anomalous transport in stressed rough fractures, Earth Planet. Sci. Lett. 454, 46 (2016).

[19] M. F. Shlesinger, B. J. West, and J. Klafter, Lévy Dynamics of Enhanced Diffusion: Application to Turbulence, Phys. Rev. Lett. 58, 1100 (1987).

[20] S. Thalabard, G. Krstulovic, and J. Bec, Turbulent pair dispersion as a continuous-time random walk, J. Fluid Mech. 755, R4 (2014).

[21] I. M. Sokolov, Models of anomalous diffusion in crowded environments, Soft Matter 8, 9043 (2012).

[22] G. Dagan, Flow and Transport in Porous Formations (Springer, New York, 1989).

[23] J. D. Hyman, S. L. Painter, H. Viswanathan, N. Makedonska, and S. Karra, Influence of injection mode on transport properties in kilometer-scale three-dimensional discrete fracture networks, Water Resour. Res. 51, 7289 (2015).

[24] A. Frampton and V. Cvetkovic, Significance of injection modes and heterogeneity on spatial and temporal dispersion of advecting particles in two-dimensional discrete fracture networks, Adv. Water Resour. 32, 649 (2009).

[25] P. K. Kang, M. Dentz, T. Le Borgne, S. Lee, and R. Juanes (unpublished).

[26] V. D. Cvetkovic, G. Dagan, and A. M. Shapiro, An exact solution of solute transport by one-dimensional random velocity fields, Stoch. Hydrol. Hydraul. 5, 45 (1991).

[27] V. Cvetkovic, H. Cheng, and X.-H. Wen, Analysis of nonlinear effects on tracer migration in heterogeneous aquifers using Lagrangian travel time statistics, Water Resour. Res. 32, 1671 (1996). 
[28] A. Baule and R. Friedrich, Joint probability distributions for a class of non-Markovian processes, Phys. Rev. E 71, 026101 (2005).

[29] P. K. Kang, M. Dentz, T. Le Borgne, and R. Juanes, Anomalous transport on regular fracture networks: Impact of conductivity heterogeneity and mixing at fracture intersections, Phys. Rev. E 92, 022148 (2015).

[30] R. Kubo, M Toda, and N. Hashitsume, Statistical Physics II, Non-Equilibrium Statistical Mechanics (Springer, Berlin, 1991).

[31] G. Margolin and B. Berkowitz, Spatial behavior of anomalous transport, Phys. Rev. E 65, 031101 (2002).

[32] M. Dentz, A. Cortis, H. Scher, and B. Berkowitz, Time behavior of solute transport in heterogeneous media: Transition from anomalous to normal transport, Adv. Water Resour. 27, 155 (2004).

[33] A. D. Polyanin and A. V. Manzhirov, Handbook of Integral Equations (CRC, Boca Raton, 1998). 